\title{
Studies on the effects of xenobiotic on radial growth and dry weight biomass of the soil fungus Trichoderma sp.
}

\author{
M.C. Freddy Vanlalmuana, R. Lalfakzuala* \\ Laboratory of Microbiology, Department of Botany, Mizoram University, Tanhril 796004, Mizoram, India \\ Corresponding author: lalfaka@yahoo.com
}

\begin{abstract}
Plant growth and development are greatly influenced by soil edaphic factor and microbial community present in the rhizosphere. Although some microorganisms are important, some can cause disease in plants and lead to the death of the plant. Trichoderma sp. is an important soil fungus which has the potential to control other soil-borne plant pathogens. To measure the bio-efficacy of Trichoderma sp. in integrated pest management, its compatibility along with other pesticides has to be assessed. GLV-71 (nitrosoglyphosate) and Elpida (emamectin benzoate) are the two commonly used herbicides and insecticides worldwide. We investigated the application of these two pesticides with their effect on the growth of Trichoderma sp. Different concentrations (half dose, field recommended dose and double dose) of the two pesticides were tested on the growth of the selected fungal species in vitro. The result shows that nitrosoglyphosate and emamectin benzoate have concentration dependent effect on the growth of Trichoderma sp. Between the two pesticides, GLV-71 shows greater effect on the growth of the microorganism. This experiment shows that Elpida exhibit more compatibility along with Trichoderma sp. as a potential bio-control agent rather than GLV-71.
\end{abstract}

Keywords: Trichoderma sp., bioefficacy, nitrosoglyphosate, emamectin benzoate, integrated pest management.

\section{INTRODUCTION}

Xenobiotics (from Greek Xenos meaning strange or foreigner) are compounds that do not occur in nature and are chemically synthesized. Such compounds are of crucial concern in the soil environment as they can influence many biological and biochemical reactions in soils. They may be grouped as antioxidants, carcinogens, drugs, environmental pollutants, food additives, hydrocarbons, and pesticides. Pesticides are chemicals used to eliminate or control a variety of agricultural pests that can damage crops and livestock and reduce farm productivity but they also have potential to affect non-target organisms and soil biochemical processes. The influence of pesticides on soil microorganisms is dependent on physical, chemical and biochemical conditions, in addition to nature and concentration of the pesticides.

Trichoderma is a fungal genus described in 1794. It includes anamorphic fungi isolated primarily from soil and decomposing organic matter (Persoon, 1794). Strains within this genus include a wide spectrum of evolutionary solutions that range from very effective soil coloniz- ers with high biodegradation potential, to non-strict plant symbionts that colonize the rhizosphere. They are facultative saprobes, and present in almost every soil type. They are known to produce an important enzyme like the cellulolytic enzymes (cellulase and hemicellulase), $\beta$-glucosidase, xylanase and chitinase. They are also an important biocontrol agent, a mycoparasites, used for controlling soil-borne fungal plant diseases (Papavizas, 1985). Mechanisms of their invasion include antibiosis, parasitism, inducing host-plant resistance, and competition. Most biocontrol agents are from the species T. harzianum, T. viride and T. hamatum. They form white, yellow-green or bright green colonies in pure culture. The conidia are borne on a short phialides on the conidiophores. This antagonistic potential is the base for effective applications of different Trichoderma strains as an alternative to the chemical control against a wide set of fungal plant pathogens (Chet, 1987; Harman and Bjorkman, 1998), and the biodegradative capacity is a source of useful enzymes in different industrial sectors (Harman and Kubicek, 1998).

Species of Trichoderma are being used either as seed 
treatment or soil application. In both the cases, the antagonist has been continuously exposed to different insecticides applied to the field either in soil or as foliar sprays. Insecticides sprayed aerially reaches the soil by means of air currents or are washed off the plant surface due to rain and is likely to influence the efficacy of native or applied biocontrol agents like Trichoderma. Hence, it is necessary to assess Trichoderma compatibility to insecticides in order to use in the integrated disease management systems.

\section{MATERIAlS AND MethodS}

The experiment is designed to show the effect of two pesticides which are commonly employed in Mizoram, viz. Elpida (emamectin benzoate) and GLV-71 (glyphosate), on Trichoderma sp. Three different concentrations of the pesticide, viz. field recommended dose, half dose and double dose, are prepared for this experiment. Trichoderma sp. is isolated from forest soil by serial dilution method which is cultured on Rose Bengal agar medium and Czapek Dox medium. Czapek Dox medium is used for stock solution.

Inhibition percentage of radial growth was measured based on control plate colony diameter using the formula (Waksman et al., 1992):

$$
\text { Mycelial growth inhibition }(\%)=\frac{A-X}{X} \times 100
$$

Where,

$\mathrm{X}=$ growth of mycelial colony in control set after incubation period subtracting the diameter of inoculum disc.

$A=$ growth of mycelial colony in treatment set after incubation period subtracting the diameter of inoculum disc. The average diameter of the fungal colonies was measured on the $3^{\text {rd }}, 4^{\text {th }}$, and $5^{\text {th }}$ day of incubation and percentage of mycelial growth inhibition was calculated.

Study was carried out by poisoned food technique using different concentrations of pesticides against Trichoderma (Jaworski, 1972). The field recommended doses of GLV-71 and Elpida are $0.437 \mathrm{~g} / 100 \mathrm{ml}$ distilled $\mathrm{H}_{2} \mathrm{O}$ and $0.050 \mathrm{~g} / 100 \mathrm{ml}$ distilled $\mathrm{H}_{2} \mathrm{O}$, respectively. By using these, half and double dose of both the pesticides are also prepared and desired volume are added to solid and liquid cultures of Trichoderma.

\section{Enumeration of fungal population}

Serial dilution and plating method (Waksman et al., 1992) was used for the isolation of fungal populations. 10 $\mathrm{g}$ of soil sample was added to $100 \mathrm{ml}$ distilled water in a $100 \mathrm{ml}$ conical flask to give 1:10 dilution. The flask was swirled for 15 minutes to get a homogenous solution. Then, $10 \mathrm{ml}$ aliquot solution was transferred to another conical flask with $90 \mathrm{ml}$ of distilled water to get 1:100 dilutions. Finally, $10 \mathrm{ml}$ of this solution was again added to $90 \mathrm{ml}$ distilled water in another flask to get 1:1000 dilutions.

Serial dilution technique:

$10 \mathrm{~g}$ of soil $+100 \mathrm{ml} \mathrm{H} \mathrm{H}_{2}(1: 10)$

$10 \mathrm{ml}$ of above solution $+90 \mathrm{ml} \mathrm{H} \mathrm{H}_{2}(1: 100)$

$10 \mathrm{ml}$ of above solution $+90 \mathrm{ml} \mathrm{H}_{2} \mathrm{O}(1: 1000)$

Rose Bengal agar medium (Martin, 1950) was used for the culture medium. Fungi were inoculated into the growth medium by adding $1 \mathrm{ml}$ of the aqueous solution $(1: 1000)$ to a plate containing the culture medium. The plates are gently rotated and the inoculums dispersed with a spreader during the addition of the aqueous solution. The plates were incubated for 7 days at $25 \pm 1^{\circ} \mathrm{C}$ in a BOD incubator.

\section{Isolation and identification of the fungal species}

The required fungal species was isolated and identified with the help of compound microscope. A thin thread of mycelia was taken out with the help of needle or pin from the mixed colony of the culture medium and it was then kept on a glass slide. The specimen is stained with cotton blue stain and it was then covered with a cover slip. The slide was then observed under compound microscope. The required fungal species Trichoderma sp. was identified and prepared for its pure culture. A portion of the colony was transferred to Czapek Dox agar medium for pure culture. After few days, the mycelium from the pure culture colony was transferred to Czapek Dox broth medium for stock culture, which was incubated for several days. Trichoderma sp. from this stock culture was used for further experiment.

\section{Preparation of different solution of pesticides}

Three different solutions of the pesticides are prepared by using the recommended rate as per labeled on the package. Based on this field recommended dose, a double and half dose of the pesticides are also prepared.

$$
\begin{array}{lr}
\text { GLV-71 } & \\
\text { Half } & -0.218 \mathrm{~g} / 100 \mathrm{ml} \text { distilled } \mathrm{H}_{2} \mathrm{O} \\
\text { Recommended }-0.437 \mathrm{~g} / 100 \mathrm{ml} \text { distilled } \mathrm{H}_{2} \mathrm{O} \\
\text { Double } & -0.875 \mathrm{~g} / 100 \mathrm{ml} \text { distilled } \mathrm{H}_{2} \mathrm{O} \\
\text { Elpida } & \\
\hline \text { Half } & -0.025 \mathrm{~g} / 100 \mathrm{ml} \text { distilled } \mathrm{H}_{2} \mathrm{O} \\
\text { Recommended }-0.050 \mathrm{~g} / 100 \mathrm{ml} \text { distilled } \mathrm{H}_{2} \mathrm{O} \\
\text { Double } & -0.100 \mathrm{~g} / 100 \mathrm{ml} \text { distilled } \mathrm{H}_{2} \mathrm{O}
\end{array}
$$

\section{Treatment of solid medium with pesticides}

The above calculated weights of different doses of the pesticides were added to $100 \mathrm{ml}$ of Czapek Dox Agar medium in six different conical flasks before it is autoclaved. The medium was then poured into Petri-plates, maintaining three replicates. Medium without pesticides treatment was also prepared for control. After the medium gets solidified, three holes are made in each plate using a cork borer. Inoculum from the stock Trichoderma 
culture is then added to the medium inside the holes with a volume of $0.4 \mathrm{ml}$. The plates are then incubated for in an incubator at $25 \pm 1^{\circ} \mathrm{C}$. These will be used for the determination of the growth rate by measuring the size of diameter of the colony. The radial growth of Trichoderma was measured on the $3^{\text {rd }}, 4^{\text {th }}$ and $5^{\text {th }}$ day from incubation. The radial growth is measured by the total diameter minus the diameter of the cork borer.

$$
\begin{aligned}
& R=2-1 \\
& \text { Where, } \\
& d 2=\text { diameter of colony } \\
& d 1=\text { diameter of cork borer. }
\end{aligned}
$$

\section{Treatment of broth medium with pesticides}

The above calculated weights of different doses of the pesticides are added to $100 \mathrm{ml}$ of Czapek Dox Broth medium in six different conical flasks before it is autoclaved. The medium is then poured into test tubes, $15 \mathrm{ml}$ each, maintaining three replicates for each dose. Medium without pesticides treatment are also prepared for control. Inoculums from the stock Trichoderma culture is then added to the test tubes with a volume of $1 \mathrm{ml}$ each. The test tubes are then incubated for 7 days in an incubator at $25 \pm 1^{\circ} \mathrm{C}$. These will be used for the determination of the growth by measuring the dry weight of the biomass. After 7 days, the biomass was filtered through Whatman filter paper no. 1 . The initial weight and the final weight of the filter were compared.

Dry weight biomass is calculated by using the formula:

Dry weight $(\mathrm{gm})=\mathrm{W} 2-\mathrm{W} 1$

Where,

W2 = final weight of filter paper

W1 = initial weight of filter paper

\section{RESULTS}

\section{Effect of pesticides on the radial growth of Tricho- derma}

The radial growth of Trichoderma from four different plates treated with different dose of the pesticides (control, half, recommended and double) were recorded on the $3^{\text {rd }}, 4^{\text {th }}$ and $5^{\text {th }}$ day from inoculation. Both the pesticides amended medium show inhibitory effect on the radial growth. GLV 71 showed more inhibitory effect on the radial growth than ELPIDA. The area of inhibition increased with the increase in the concentration of the pesticides. Maximum size of colony is found in control plate with $4.22 \pm 0.061 \mathrm{~cm}$ after 3 days, $8.07 \pm 0.130 \mathrm{~cm}$ after 4 days and $12.97 \pm 0.063 \mathrm{~cm}$ after 5 days of incubation. Among the plate treated with GLV-71 - 80.52\%, $95.78 \%$ and $100 \%$ inhibition are shown in half, recommended and double dose plate respectively after 3 days of incubation; $75.24 \%, 88.72 \%$ and $97.38 \%$ inhibition in half, recommended and double dosages after 4 days; $70.53 \%, 82.95 \%$ and $93.23 \%$ inhibition in half, recommended and double plate after 5 days. Among the plate treated with ELPIDA - 54.99\%, 74.1\%, 84.21\% inhibition in half, recommended, double plates after 3 days; $62.44 \%, 68.22 \%, 80.05 \%$ inhibition in half, recommended, double after 4 days; $66.34 \%, 71.56 \%, 77.38 \%$ inhibition in half, recommended, double dose after 5 days of incubation. The one-way ANOVA result shows a significant variation in the different size of the colony as shown in Figures 1 \& 2.

\section{Effect of pesticides on the dry weight biomass of Trichoderma $s p$.}

Mycelium dry weight of Trichoderma sp. was measured from four different treatments, viz. control, half, recommended and double dose of GLV-71 and Elpida respectively. Control culture has maximum dry weight of $0.388 \pm 0.016 \mathrm{~g}$ after 3 days of incubation. Among the cultures treated with GLV-71, dry weight of $0.182 \pm 0.019$ $\mathrm{g}$ is found in culture mixed with half dose, $0.100 \pm 0.023 \mathrm{~g}$ in culture mixed with recommended dose and $0.040 \pm$ $0.019 \mathrm{~g}$ in culture with double dose. In cultures treated with Elpida, dry weight of $0.205 \pm 0.027 \mathrm{~g}$ is found in culture mixed with half dose, $0.138 \pm 0.007 \mathrm{~g}$ in culture treated with recommended dose and $0.059 \pm 0.030 \mathrm{~g}$ in culture treated with double dose. The one-way ANOVA results show a significant variation in the different size of the colony as shown in Figure 3.

\section{DISCUSSION}

The influence of pesticides on soil microorganisms is dependent on physical, chemical and biochemical conditions, in addition to nature and concentration of the pesticides. The use of pesticides on agricultural field has also shown effect on non-target microorganisms. Such microorganisms are of great importance for the plants in many ways. Many pesticides have been used to study their effect on the population of soil micro biota.

In this experiment, two pesticides GLV-71 (herbicide) and Elpida (insecticide) were used to study their effect on Trichoderma sp. The present study reveals that these pesticides affect the growth rate of Trichoderma sp. and reduce production of spores with the increasing concentration of the pesticides.

Glyphosate is the active ingredient of several broadspectrum commercial herbicides, which are translocated from leaves to roots and act by blocking the shikimic acid pathway in plants (Johal and Rahe, 1984; Steinruncken and Amrhein, 1980; Boocock and Coggins, 1983). Unlike the effect of herbicides on plants, very little is known about the mode of action of glyphosate on fungi. Glyphosate blocks the shikimic acid pathway in Neurospora crassa and presumable, could have the same effect in 
Figure 1: Effect of GLV-71 on radial growth Trichoderma sp.

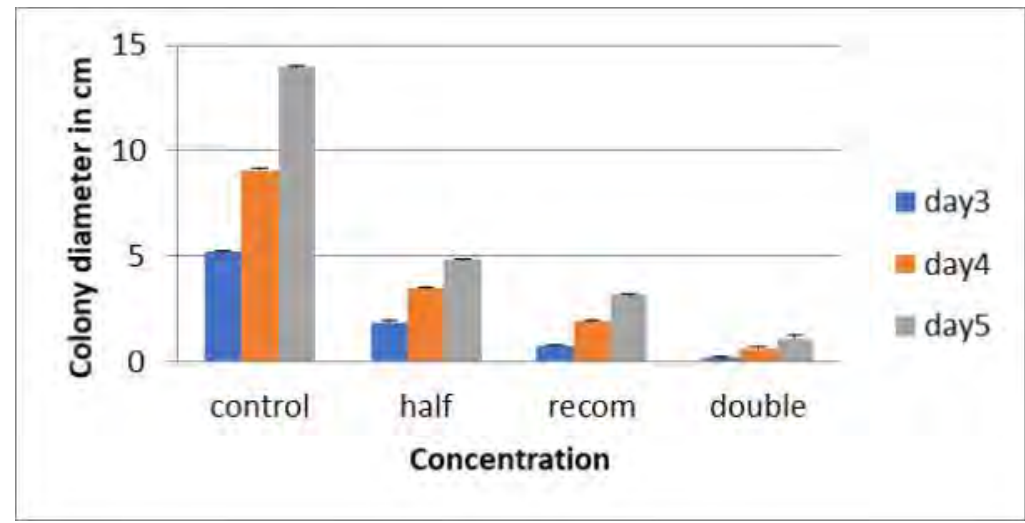

Figure 2: Effect of Elpida on radial growth of Trichoderma sp.

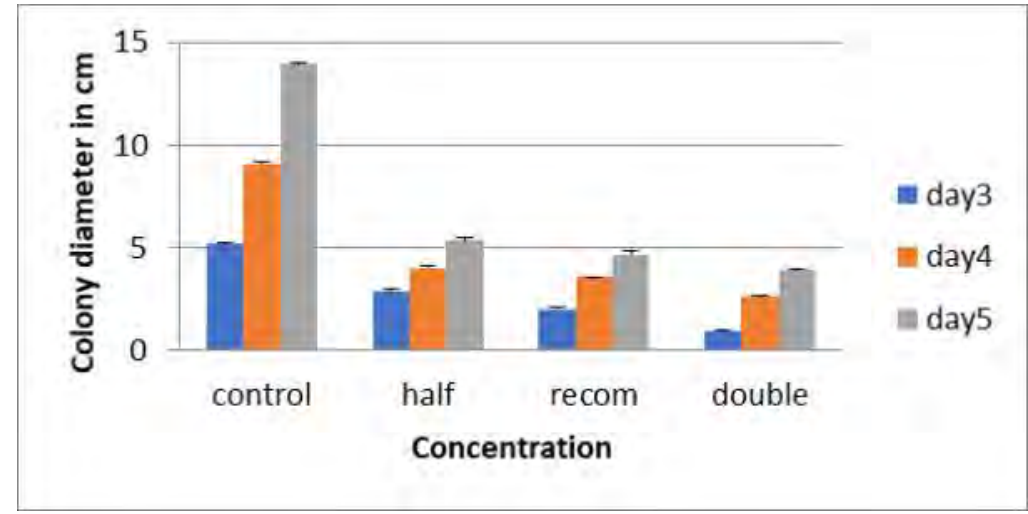

Figure 3: Effect of GLV-71 and Elpida on dry weight biomass of Trichoderma sp.

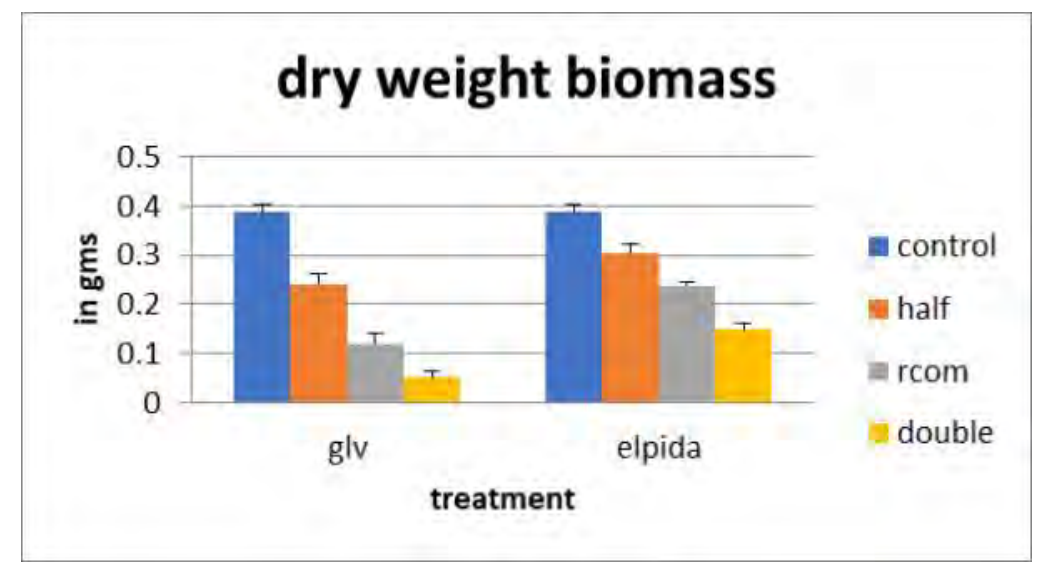

other fungal species (Ranganathswamy et al., 2011).

Observations made on the radial growth of Trichoderma sp. indicated significant variation in the sensitivity of Trichoderma sp. isolate to pesticides or toxicity of pesticides towards Trichoderma isolates. When observations were recorded on radial growth for three consecutive days $\left(3^{\text {rd }}, 4^{\text {th }}\right.$, and $5^{\text {th }}$ day) in pesticides amended plates, the growth was significantly inhibited on the recorded three days of incubation. GLV-71 shows more inhibition on the growth of Trichoderma sp. than Elpida. Change in the concentration of commercial formulation of the pesticides also shows significant effect on the growth.

This finding suggests that Trichoderma sp. is more resistant to Elpida than GLV-71. This agrees with the find- ings that new generation insecticides, viz. spinosad, emamectin benzoate, thiamethoxam and indoxacarb were highly compatible with both the Trichoderma isolates with less toxic effect on the radial growth. Thus, the potential biocontrol agent Trichoderma shows more compatibility with Elpida than GLV-71.

\section{REFERENCES}

Boocock, M.R., Coggins, J.R. (1983). Kinetics of 5enolpyruvylshikimate-3-phosphate synthase inhibition by glyphosate. FEBS Letter 154: 127-133.

Chet, I. (1987). Trichoderma - application, mode of action and potential as a biocontrol agent of soilborne 
plant pathogenic fungi. In: Innovative Approaches to Plant Disease Control. I. Chet (ed.). John Wiley and Sons, New York, pp. 137-160.

Harman, G.E., Bjorkman, T. (1998). Potential and existing uses of Trichoderma and Gliocladium for plant disease control and plant growth enhancement. In: Kubicek CP, Harman GE (eds). Trichoderma and Gliocladium Vol. 2. Taylor and Francis, London, pp. 229-265.

Harman, G.E., Kubicek, P.K. (1998). Trichoderma and Gliocladium Vol 2. Enzymes, biological control and commercial applications. Taylor and Francis, London, pp. 1-393.

Jaworski, E.G. (1972). Mode of action of nphosphonomethylglycine: Inhibition of aromatic amino acid biosynthesis. J Agr Food Chem 20: 11951205.

Johal, G.S., Rahe, J.E. (1984) Effect of soil-borne plantpathogenic fungi on the herbicidal action of glyphosate on bean seedlings. Phytopathology 74: 950955.

Nene, Y.L., Thapliyal, P.N. (1993). Fungicides in Plant Disease Control ( $3^{\text {rd }}$ edition). Oxford and IBH Publishing Co. Pvt. Ltd.
Papavizas, G.C. (1985). Trichoderma and Gliocladium: biology, ecology, and potential for biocontrol: Annual Review. Phyto., 23, 23-54.

Persoon, C.H. (1794). Disposita methodica fungorum. Romer's Neues Mag Bot. 1: 81-128.

Ranganathswamy, M., Patibanda, A.K., Chandrashekar, G.S., Mallesh, S.B., Sandeep, D., Kumar, H.B. (2011). Compatibility of Trichoderma isolates to selected insecticides in vitro. Asian Journal of Bio Science, 6: 238 -240 .

Steinruncken, H.C, Amrhein, N. (1980) The herbicide glyphosate is a potent inhibitor of 5-phosphate synthase. Biochem Biophys Res Commun 94: 1207-1212.

Sundar, A.R., Das, N.D., Krishnaveni, D. (1995). In-vitro antagonism of Trichoderma spp. against two fungal pathogens of Castor. Indian J Plant Protect 23: 152155.

Waksman, G., Kominos, D., Robertson, S.R., Pant, N., Baltimore, D., Birge, R.B., Cowburn, D., Hanafusa, H., Mayer, B.J., Overduin, M., Resh, M.D., Rios, C.B., Silverman, L., Kuriyan, J. (1992). Crystal structure of the phosphotyrosine recognition domain $\mathrm{SH} 2$ of $\mathrm{v}$-src complexed with tyrosine-phosphorylated peptides. Nature, 358, 646-653. 